\title{
Past history of stage I/II solid tumor malignancy impacts considerably on sepsis mortality: a propensity score matching analysis from the hellenic sepsis study group
}

\author{
George Dimopoulos ${ }^{1}$, Nikoletta Rovina ${ }^{2}$, Maria Patrani ${ }^{3}$, Eleni Antoniadou ${ }^{4}$, Dimitrios Konstantonis ${ }^{1}$, \\ Konstantina Vryza ${ }^{5}$, Glykeria Vlachogianni ${ }^{6}$, Miltiades Kyprianou ${ }^{7}$, Christina Routsi ${ }^{8}$, \\ Evangelos J. Giamarellos-Bourboulis ${ }^{79^{*}}$ (i) and on behalf of the Hellenic Sepsis Study Group
}

\begin{abstract}
Background: Whether past history of solid stage I/II inactive cancer has an impact on 28-day mortality of sepsis remains unclear. We aimed to determine the impact of history of stage I or II solid tumor malignancy in complete remission the last 3 years on sepsis outcome.

Methods: Using the database of the Hellenic Sepsis Study Group from 1553 patients with sepsis admitted in the ICU, 83 patients with sepsis by Sepsis-3 definition with past-history of stage I/II inactive solid malignancy the last 3 years were depicted. A comparator group of 83 patients fully matched for age, severity, type of infection and comorbidities was selected by propensity score matching.

Results: Mortality after 28 days was $37.3 \%$ in the comparator group and $54.2 \%$ in the solid tumor stage I/II group (odds ratio for death 1.98; p: 0.030). Following step-wise forward Cox regression analysis, septic shock (hazard ratio 1.80), acute renal injury (hazard ratio 2.06), history of coronary heart disease (hazard ratio 0.36) and history of stage $1 / 1 /$ solid tumor malignancy (hazard ratio 1.79) were the only independent variables associated with 28-day mortality. Serum levels of procalcitonin and of soluble urokinase plasminogen activator receptor were similar between the two groups of comparisons.
\end{abstract}

Conclusions: Past history of stage I/II solid malignancy is an independent risk factor for unfavorable outcome from sepsis the first 28 days.

Keywords: Cancer, ICU, Severity, Outcome

\section{Background}

Throughout the past decades the low survival rates of critically ill patients suffering from malignancies led clinicians to the decision to avoid admission of these patients in the Intensive Care Unit (ICU) [1]. The advances

\footnotetext{
*Correspondence: egiamarel@med.uoa.gr

${ }^{7} 4$ th Department of Internal Medicine, National and Kapodistrian University of Athens, Medical School, Athens, Greece

${ }^{9} 4$ th Department of Internal Medicine, ATTIKON University Hospital, 1 Rimin Street, 12462 Athens, Greece

Full list of author information is available at the end of the article
}

in early detection and management of hematological malignancies and solid tumors over the last years have resulted in an increased number of ICU admissions of cancer patients who had significantly lower mortality in comparison to the 1980's and the 1990's [2, 3]. Large multicenter studies have shown that these patients account for $15-20 \%$ of all ICU admissions [4, 5]. The main causes of ICU admission do not differ between patients with malignancies and the general population [6] with sepsis being the second most frequent reason

(C) The Author(s). 2019 Open Access This article is distributed under the terms of the Creative Commons Attribution 4.0 International License (http://creativecommons.org/licenses/by/4.0/), which permits unrestricted use, distribution, and reproduction in any medium, provided you give appropriate credit to the original author(s) and the source, provide a link to the Creative Commons license, and indicate if changes were made. The Creative Commons Public Domain Dedication waiver (http://creativecommons.org/publicdomain/zero/1.0/) applies to the data made available in this article, unless otherwise stated. 
for ICU admission of cancer patients following postoperative care [5].

It is largely conceived that cancer patients are more prone to sepsis and unfavorable outcome due to factors associated with their malignancies like chemotherapyinduced immunosuppression and metastasis-associated organ obstructions [7]. However, no data are available if history of cancer that remains inactive may per se become a comorbidity driving towards unfavorable outcome.

In order to reply to this question, we used the database of the Hellenic Sepsis Study Group (HSSG) (www.sepsis. gr) that includes a broad collection of clinical data from both non-ICU and ICU patients with sepsis. We aimed to analyze the impact of history of stage I or II solid tumor malignancies in complete remission the last 3 years on the 28-day mortality of patients with sepsis admitted in the ICU.

\section{Methods \\ Patients}

The HSSG is collecting since June 2006 clinical data of patients with clinically and/or microbiologically documented infections and systemic inflammatory response (SIRS) from 55 departments across Greece; 21 study sites are ICUs. This is done by a central protocol that has been approved by the Ethics Committees of the participating hospitals where the study sites are located. Adult patients are enrolled after written informed consent provided by the patients or first degree relatives in the case of patients unable to consent. Since March 2016 all patients in the database were re-classified into infection, sepsis and septic shock using the Sepsis-3 classification criteria [8]. Patients with febrile neutropenia and infection by the human immunodeficiency virus are excluded from the HSSG registry.

In January 2019, it was decided to investigate the role of history of inactive stage I/II lung and colorectal adenocarcinoma in the final outcome of sepsis among patients admitted for sepsis in the ICU. Investigators from all ICUs participating in the HSSG defined specific criteria for the selection of patients of the database for this analysis. These criteria were: a) admission in the ICU; b) sepsis defined by Sepsis-3 definition; c) history of stage I or II solid tumor in complete remission the last 3 years that did not predispose to infection; and d) lung cancer and colorectal cancer. Exclusion criteria were: a) any solid tumor malignancy of stages III or IV; b) any hematologic malignancy; c) any lymphoma; d) intake of chemotherapy; e) ICU admission due to postoperative care for cancer resection; and f) malignancy as direct predisposition to sepsis. The last exclusion criterion comprised, but was not limited, to the following conditions: acute ascending cholangitis due to biliary tract obstruction by tumor; acute ascending pyelonephritis due to ureter obstruction by tumor; lung infection due to bronchial obstruction by tumor; and intraabdominal infection developing after tumor resection.

Patients remaining after the application of the inclusion and exclusion criteria were divided into two groups; those with stage I/II solid tumor malignancy and comparators without any malignancy. Two fully matched groups were formatted with an equal number of patients using specific matching criteria. These criteria were age, SOFA score, APACHE II score, Charlson's Comorbidity Index (CCI), presence of septic shock and type of infection. Matching was done by propensity score calculation.

Blood was sampled from all enrolled patients within the first $24 \mathrm{~h}$ from enrolment. Blood was collected into sterile and pyrogen-free and anticoagulant-free tubes (Vacutainer, Becton Dickinson, Cockeysville Md). Tubes were transported within 1 day by a courier service to the Laboratory of Immunology of Infectious Diseases of the 4th Department of Internal Medicine at ATTIKON University Hospital of Athens. Tubes were centrifuged and serum was kept refrigerated at $-70{ }^{\circ} \mathrm{C}$ until assayed. Procalcitonin (PCT) was estimated in serum in duplicate by an immuno-time-resolved amplified cryptate technology assay (Kryptor PCT; BRAHMS GmbH, Henningdorf, Germany) with a functional assay sensitivity of $0.06 \mathrm{ng} /$ $\mathrm{ml}$. Concentrations of soluble urokinase plasminogen activator receptor (suPAR) were measured by an enzyme immunosorbent assay with a functional assay sensitivity of $2.1 \mathrm{ng} / \mathrm{ml}$ (suPARnostic, Virogates, Medicon Valley, Denmark).

The following information was recorded per patient: demographics, severity using the APACHE II score and SOFA score, comorbidities, microbiology, and administered antimicrobials. Patients were followed-up for 28 days. The selection and dose of administered antimicrobials for all patients was done according to the national guidelines that are published by the Hellenic Society for Infectious Diseases (www.loimoxeis.gr). The collection of data in each study site was confirmed by independent monitors.

\section{Study endpoints}

The primary study endpoint was to determine whether history of stage I/II cancer is influencing 28-day mortality of patients with sepsis who are hospitalized in the ICU. The secondary endpoint was to define if there are differences in the concentrations of PCT and suPAR between the two groups.

\section{Statistical analysis}

Comparisons between comparators and patients with history of stage I/II solid malignancy were done by the Fisher exact test for binary variables, by the Student's t- 
test for parametric continuous variables and by the Mann-Whitney $U$ test for non-parametric continuous variables. Survival was compared by the log-rank test. The odds ratio (OR) and 95\% confidence intervals (CIs) were determined by the Mantel and Haenszel test. Variables differing significantly between survivors and nonsurvivors entered the equation of forward step-wise Cox regression analysis as dependent variables with 28-day mortality as the independent variables. Hazard ratios and 95\% CIs were calculated. Concentrations of PCT and suPAR were expressed as median and 95\% CIs. Any $p$-value below 0.05 was considered significant. Analysis was done using IBM SPSS statistics version 25.0.

\section{Results}

The study flow chart is shown in Fig. 1. Using propensity score matching, two groups were formatted; comparators without malignancy $(n=83)$ and patients with history of stage I/II solid malignancy $(n=83)$. The two groups were fully matched for APACHE II score, SOFA score, CCI, age and organ dysfunctions. The time to the administration of the first dose of antimicrobials from the onset of sepsis or vasopressors did not differ between groups. Microbiological confirmation of the pathogen was available in 86 patients. Susceptibilities of the isolated pathogens to the administered antimicrobials did not differ between the two groups (Table 1).

Mortality after 28 days was $37.3 \%$ in the comparator group (31 out of 83 patients) and $54.2 \%$ in the solid tumor stage I/II group (45 out of 83) (Fig. 2). The OR for death among patients with history of solid tumor was 1.98 (95\%CIs: 1.07-3.69; p: 0.030). Nil patients in the malignancy group died due to cancer.

At a next step, baseline demographics were compared between 28-day survivors and non-survivors. Variables being significantly different between the two groups of comparisons were septic shock, acute kidney and history of stage I/II solid malignancy that were more common among non-survivors; and history of coronary heart disease that was more common among survivors (Table 2). The impact of these four variables was confirmed after forward step-wise Cox regression analysis (Table 3). Analysis was repeated for these variables among the 86 patients with microbiologically confirmed infections. Susceptibility of the pathogen to the administered antimicrobials was included as another variable in

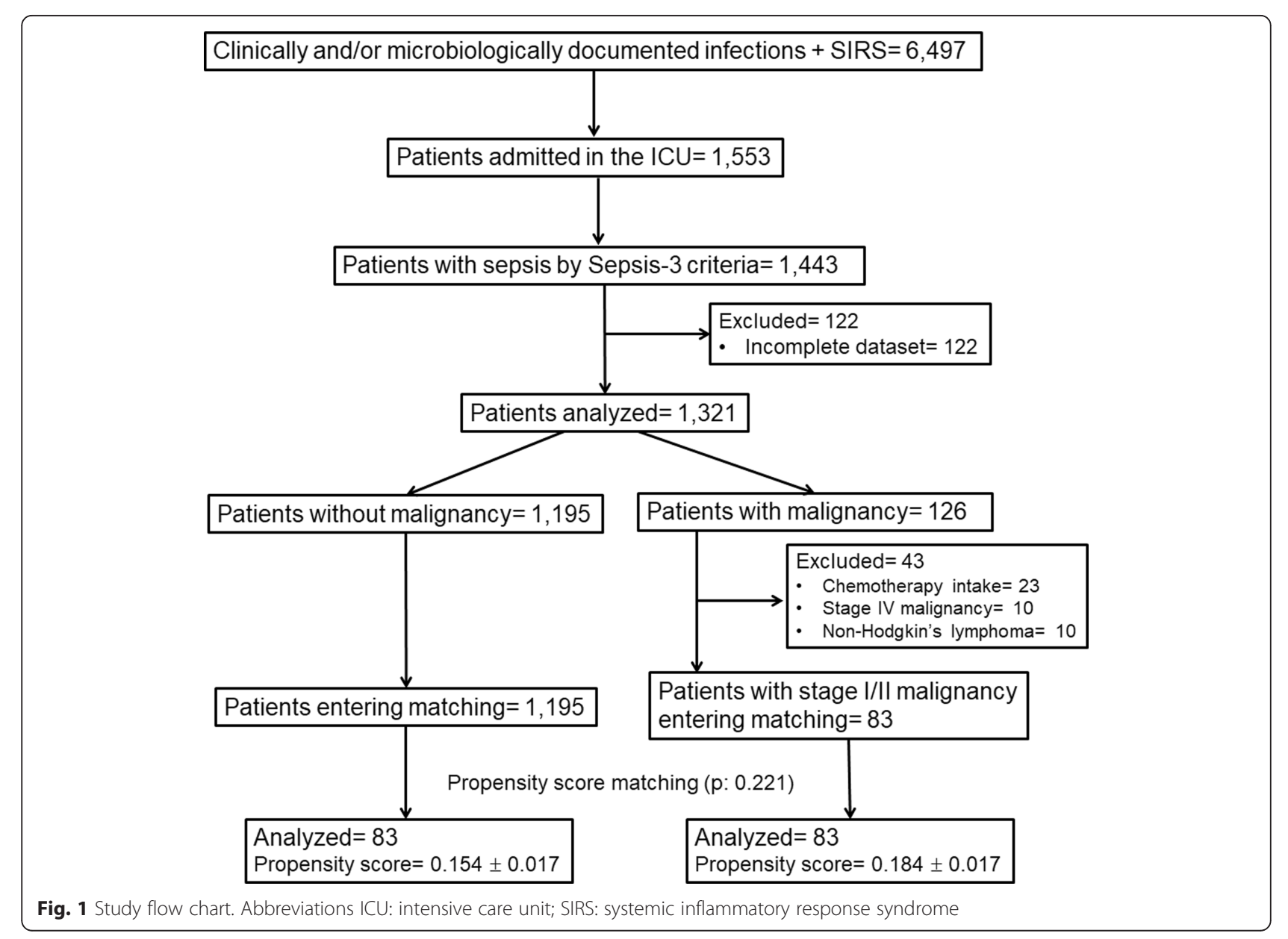


Table 1 Characteristics of analyzed patients

\begin{tabular}{|c|c|c|c|}
\hline & $\begin{array}{l}\text { Sepsis without } \\
\text { malignancy }(n=83)\end{array}$ & $\begin{array}{l}\text { Sepsis with stage } 1 / \| \text { solid } \\
\text { malignancy }(n=83)\end{array}$ & $p$-value \\
\hline Male gender (n, \%) & $56(67.5)$ & $53(63.9)$ & 0.744 \\
\hline Age (years, mean $\pm S D$ ) & $64.0 \pm 18.4$ & $69.10 \pm 9.85$ & 0.435 \\
\hline APACHE II score (mean \pm SD) & $21.2 \pm 8.1$ & $21.9 \pm 6.9$ & 0.782 \\
\hline SOFA score (mean \pm SD) & $8.47 \pm 3.74$ & $8.52 \pm 3.39$ & 0.915 \\
\hline $\mathrm{CCl}($ mean $\pm \mathrm{SD})$ & $4.70 \pm 3.37$ & $5.64 \pm 2.27$ & 0.057 \\
\hline White blood cells $\left(/ \mathrm{mm}^{3}\right.$, mean $\left.\pm \mathrm{SD}\right)$ & $15,783.3 \pm 8868.6$ & $14,121.2 \pm 8977.1$ & 0.120 \\
\hline \multicolumn{4}{|l|}{ Infection site (n, \%) } \\
\hline Hospital-acquired pneumonia & $26(31.3)$ & $25(30.1)$ & 1.00 \\
\hline Primary bacteremia & $15(18.1)$ & $15(18.1)$ & 1.00 \\
\hline Intrabdominal infection & $14(16.9)$ & $21(25.3)$ & 0.253 \\
\hline Community-acquired pneumonia & $14(16.9)$ & $10(12.0)$ & 0.509 \\
\hline Acute pyelonephritis & $5(6.0)$ & $3(3.6)$ & 0.720 \\
\hline Acute bacterial skin and soft tissue infection & $4(4.8)$ & $0(0)$ & 0.120 \\
\hline Bacterial meningitis & $1(1.2)$ & $1(1.2)$ & 1.00 \\
\hline Other & $4(4.8)$ & $8(9.6)$ & 0.369 \\
\hline \multicolumn{4}{|l|}{ Organ dysfunction (n, \%) } \\
\hline Septic shock & $56(67.5)$ & $56(67.5)$ & 1.00 \\
\hline Acute respiratory distress syndrome & $44(53.0)$ & $46(55.4)$ & 0.876 \\
\hline Acute kidney injury & $22(26.5)$ & $12(14.5)$ & 0.083 \\
\hline Disseminated intravascular coagulation & $17(20.5)$ & $22(26.5)$ & 0.464 \\
\hline \multicolumn{4}{|l|}{ Bloodstream isolate (n, \%) } \\
\hline Acinetobacter baumannii & $7(8.4)$ & $9(10.8)$ & 0.793 \\
\hline Klebsiella pneumoniae & $3(3.6)$ & $5(6.0)$ & 0.720 \\
\hline Pseudomonas aeruginosa & $3(3.6)$ & $5(6.0)$ & 0.720 \\
\hline Staphylococcus aureus & $3(3.6)$ & $0(0)$ & 0.245 \\
\hline Enterococcus faecalis & $3(3.6)$ & $3(3.6)$ & 1.00 \\
\hline Enterococcus faecium & $1(1.3)$ & $3(3.6)$ & 0.621 \\
\hline Other & $9(10.8)$ & $6(7.2)$ & 0.600 \\
\hline \multicolumn{4}{|l|}{ Urine isolate $(n, \%)$} \\
\hline Klebsiella pneumoniae & $2(2.4)$ & $2(2.4)$ & 1.00 \\
\hline Acinetobacter baumannii & $3(3.6)$ & $1(1.3)$ & 0.620 \\
\hline \multicolumn{4}{|c|}{ Tracheobronchial secretions isolate (quantity $>10^{5} \mathrm{cfu} / \mathrm{ml}$ ) } \\
\hline Klebsiella pneumoniae & $4(4.8)$ & $8(9.6)$ & 0.370 \\
\hline Pseudomonas aeruginosa & $6(7.2)$ & $7(8.4)$ & 1.00 \\
\hline Acinetobacter baumannii & $14(16.9)$ & $22(26.5)$ & 0.187 \\
\hline Staphylococcus aureus & $3(3.6)$ & $0(0)$ & 0.245 \\
\hline Other & $6(7.2)$ & $4(4.8)$ & 0.746 \\
\hline \multicolumn{4}{|l|}{ Co-morbidities (n, \%) } \\
\hline Type 2 diabetes mellitus & $22(26.5)$ & $16(19.3)$ & 0.356 \\
\hline Chronic heart failure & $23(27.7)$ & $18(21.7)$ & 0.472 \\
\hline Chronic obstructive pulmonary disease & $17(20.5)$ & $17(20.5)$ & 1.00 \\
\hline Chronic renal disease & $11(13.3)$ & $6(7.2)$ & 0.306 \\
\hline Coronary heart disease & $12(14.5)$ & $11(3.3)$ & 1.00 \\
\hline
\end{tabular}


Table 1 Characteristics of analyzed patients (Continued)

\begin{tabular}{|c|c|c|c|}
\hline & $\begin{array}{l}\text { Sepsis without } \\
\text { malignancy }(n=83)\end{array}$ & $\begin{array}{l}\text { Sepsis with stage } 1 / / I \text { solid } \\
\text { malignancy }(n=83)\end{array}$ & $p$-value \\
\hline Vascular hypertension & $18(21.7)$ & $21(25.3)$ & 0.715 \\
\hline Atrial fibrillation & $9(10.8)$ & $8(9.6)$ & 1.00 \\
\hline Stroke & $13(15.7)$ & $10(12.0)$ & 0.654 \\
\hline $\begin{array}{l}\text { Time (hours) from sepsis onset to the administration of the first dose of } \\
\text { antimicrobials (median, range) }\end{array}$ & $5(0-125)$ & $6(0-216)$ & 0.112 \\
\hline $\begin{array}{l}\text { Time (hours) from onset of vasopressors to the administration of the first } \\
\text { dose of antimicrobials (median, range) }\end{array}$ & $2.7(0-55)$ & $2.8(0-168)$ & 0.444 \\
\hline $\begin{array}{l}\text { Susceptibility of the isolated pathogen to the administered antimicrobials } \\
\text { based on antibiogram }(n, \%)\end{array}$ & $22(26.5)$ & $22(26.5)$ & 1.00 \\
\hline
\end{tabular}

the equation. History of stage I/II solid malignancy was again confirmed to be an independent variable associated with unfavorable 28-day outcome (Table 4).

No differences in PCT and suPAR concentrations were found between the two groups of comparison (Fig. 3).

\section{Discussion}

The present study used a unique study design and tried to provide answers to a common medical question that remains unanswered. The medical question is whether past-history of stage I/II solid tumor malignancy that remains inactive impacts on 28-day mortality. Findings were affirmative but in order to answer this question an extensive filtering process was needed. During this process, only patients without treatment for their malignancy and without any apparent role of the malignancy as a factor predisposing to sepsis were analyzed. They were compared to patients matched not only for age but also for severity, organ dysfunction, type of infection and comorbidities. A similar approach has never been done before.

Available studies that investigate the outcome of patients with cancer and sepsis enroll only patients suffering from cancers and they do not narrow their analysis on stage I/II patients. Instead they enroll all patients irrespective of the presence or not of metastasis, of the intake or not of chemotherapy and of the presence of neutropenia. Analysis ends-up that 28-day mortality is dependent on the severity of organ failures where neutropenia post-chemotherapy is another independent prognostic variable for unfavorable outcome [5, 9-12]. This is in line with the results of our Cox regression analysis showing organ dysfunction as a salient risk factor for unfavorable outcome. However, most of patients with malignancy bear the risk for infections by multidrug-resistant bacteria probably due to frequent

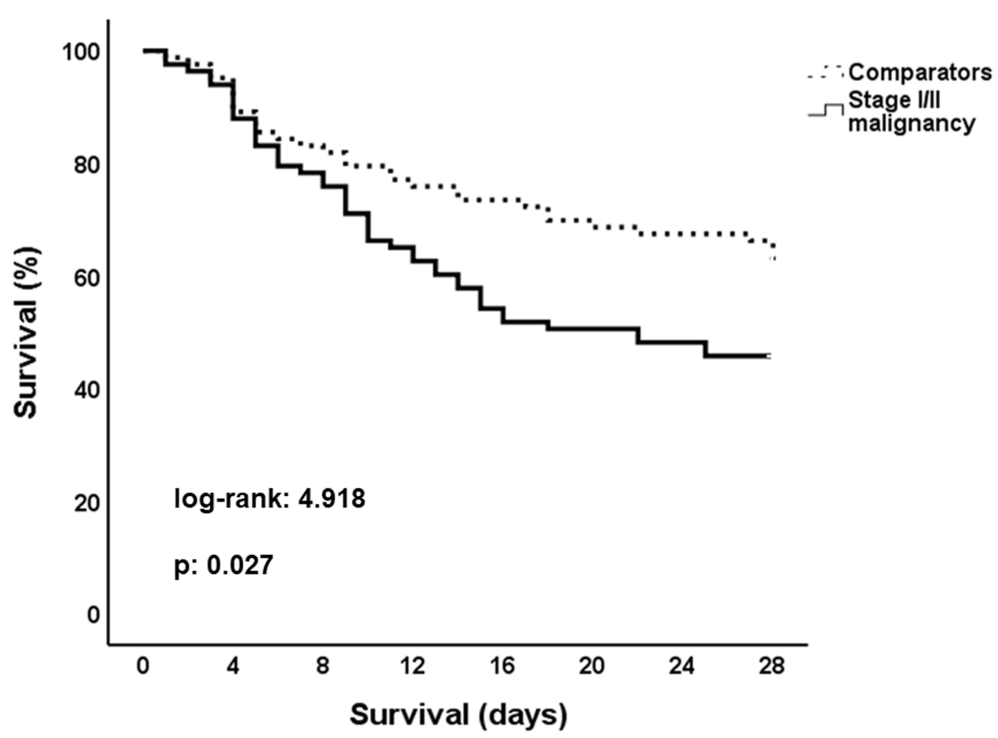

Fig. 2 Survival of 83 patients with sepsis and history of stage I/II malignancy and of 83 fully-matched sepsis comparators. The log-rank test of comparison and the respective $p$-value are provided 
Table 2 Differences in baseline characteristics between survivors and non-survivors

\begin{tabular}{|c|c|c|c|}
\hline & Survivors $(n=90)$ & Non-survivors $(n=76)$ & $p$-value \\
\hline \multicolumn{4}{|l|}{ Infection site $(n, \%)$} \\
\hline Hospital-acquired pneumonia & $27(30.0)$ & $24(31.6)$ & 1.00 \\
\hline Primary bacteremia & $13(14.4)$ & $17(22.4)$ & 0.226 \\
\hline Intrabdominal infection & $14(16.9)$ & $21(25.3)$ & 0.253 \\
\hline Community-acquired pneumonia & $12(13.3)$ & $12(15.8)$ & 0.665 \\
\hline Acute pyelonephritis & $6(6.7)$ & $2(2.6)$ & 0.291 \\
\hline Acute bacterial skin and soft tissue infection & $4(4.4)$ & $0(0)$ & 0.126 \\
\hline Bacterial meningitis & $2(2.2)$ & $0(0)$ & 0.501 \\
\hline Other & $3(3.3)$ & $0(0)$ & 0.251 \\
\hline \multicolumn{4}{|l|}{ Organ dysfunction (n, \%) } \\
\hline Septic shock & $52(57.8)$ & $60(78.9)$ & 0.005 \\
\hline Acute respiratory distress syndrome & $43(47.8)$ & $47(61.8)$ & 0.086 \\
\hline Acute kidney injury & $12(13.3)$ & $22(28.9)$ & 0.020 \\
\hline Disseminated intravascular coagulation & $17(18.9)$ & $22(28.9)$ & 0.144 \\
\hline \multicolumn{4}{|l|}{ Co-morbidities (n, \%) } \\
\hline Type 2 diabetes mellitus & $21(23.3)$ & $17(22.4)$ & 1.00 \\
\hline Chronic heart failure & $21(23.3)$ & $20(26.3)$ & 0.719 \\
\hline Chronic obstructive pulmonary disease & $16(17.8)$ & $18(23.7)$ & 0.440 \\
\hline Chronic renal disease & $9(10.0)$ & $8(10.5)$ & 1.00 \\
\hline Coronary heart disease & $18(20.0)$ & $5(6.6)$ & 0.014 \\
\hline Vascular hypertension & $23(25.6)$ & $16(21.1)$ & 0.583 \\
\hline Atrial fibrillation & $11(12.2)$ & $6(7.9)$ & 0.445 \\
\hline Stroke & $12(13.3)$ & $11(14.5)$ & 1.00 \\
\hline History of stage $1 /$ /I solid malignancy & $38(42.2)$ & $45(59.2)$ & 0.043 \\
\hline
\end{tabular}

hospitalizations leading to colonization by that species [13]. It is evident that this has a major impact on 28-day outcome through the intake of inappropriate antimicrobial therapy $[13,14]$.

Two major limitations of the study need to be addressed; matching for appropriateness of antimicrobial therapy and quality of care between study sites. Regarding administered antimicrobials therapy it needs to be

Table 3 Forward step-wise Cox regression analysis of variables associated with 28-day mortality

\begin{tabular}{llll}
\hline Variable & $\begin{array}{l}\text { Hazard } \\
\text { ratio }\end{array}$ & $\begin{array}{l}\text { 95\% confidence } \\
\text { intervals }\end{array}$ & $p$-value \\
\hline Septic shock & 1.80 & $1.01-3.22$ & 0.046 \\
$\begin{array}{l}\text { Acute kidney injury } \\
\begin{array}{l}\text { History of coronary heart } \\
\text { disease }\end{array}\end{array}$ & 0.36 & $1.21-3.49$ & $0.007-0.89$ \\
$\begin{array}{l}\text { History of stage I/II solid } \\
\text { malignancy }\end{array}$ & 1.79 & $1.13-2.85$ & 0.028 \\
\hline
\end{tabular}

Variables significantly different between survivors and non-survivors of Table 2 entered the equation as dependent variables. Variables remaining significant after four steps of analysis are shown outscored that although not all variables were addressed, patients with history of stage I/II solid tumor malignancy and comparators did not differ in timing of administration and susceptibilities of the isolated pathogens to the administered antimicrobials.

There are two more studies that are trying to address the same question but that have not succeeded to provide comparators with similar severity and comorbidities. The first study is coming from Lebanon and it is retrospective analysis of 176 oncological patients with active hematologic or solid tumor malignancy on chemotherapy or radiation therapy. These were compared to another 176 non-oncological patients coming from the same database. Although the hospital mortality of the oncological patients was significantly greater than the non-oncological patients, their severity as defined by their vital signs and requirement for vasopressors was also significantly greater than the non-oncological patients [15]. The second study is a retrospective analysis of the REGARDS database. Cancer survivors were compared to patients without cancer history with regards to hospital death and to the time until the first sepsis event. Cancer 
Table 4 Cox regression analysis of variables associated with 28day mortality among patients with microbiologically confirmed infections

\begin{tabular}{llll}
\hline Variable & $\begin{array}{l}\text { Hazard } \\
\text { ratio }\end{array}$ & $\begin{array}{l}95 \% \\
\text { confidence } \\
\text { intervals }\end{array}$ & -value \\
\hline Septic shock & 1.45 & $0.67-3.15$ & 0.345 \\
Acute kidney injury & 2.06 & $0.94-4.55$ & 0.073 \\
History of coronary heart disease & 0.74 & $0.25-2.19$ & 0.587 \\
$\begin{array}{l}\text { Susceptibility of the pathogen to the } \\
\text { administered antimicrobials }\end{array}$ & 0.54 & $0.26-1.11$ & 0.096 \\
History of stage I/ll solid malignancy & 2.72 & $1.37-5.40$ & 0.004 \\
\hline
\end{tabular}

Variables described in Table 3 entered the equation along with susceptibility of the isolated pathogen to the administered antimicrobials

survivors were defined as patients without need of chemotherapy or radiation the past 2 years. Results showed that the time to the first sepsis event was significantly shorter among cancer survivors and that in the case of infection hospital mortality was $8.29 \%$ among cancer survivors and
3.93\% among non-cancer comparators. However, the two groups were not adequately matched since cancer survivors were older and they were presenting with significantly more comorbidities than the comparators. Moreover, the analysis did not exclude patients with stage III/IV malignancies [16].

The mechanism explaining why a past history of stage I/II solid tumor malignancy predisposes to death in case of sepsis cannot be derived by our findings. It may be argued that cancer survivors are more immune debilitated due to either cancer itself or to previous cancer treatment. This is compatible with the difference of the survival curves between cases and comparators of our study starting to separate by day 7 . It is remarkable that measured PCT and SUPAR are similar with comparators showing that in these patients biomarkers need to be interpreted as in the non-cancer populations. It needs to be underscored that suPAR is a non-specific biomarker of mortality irrespective of causality [17]. Although suPAR is early increased among cancer patients with
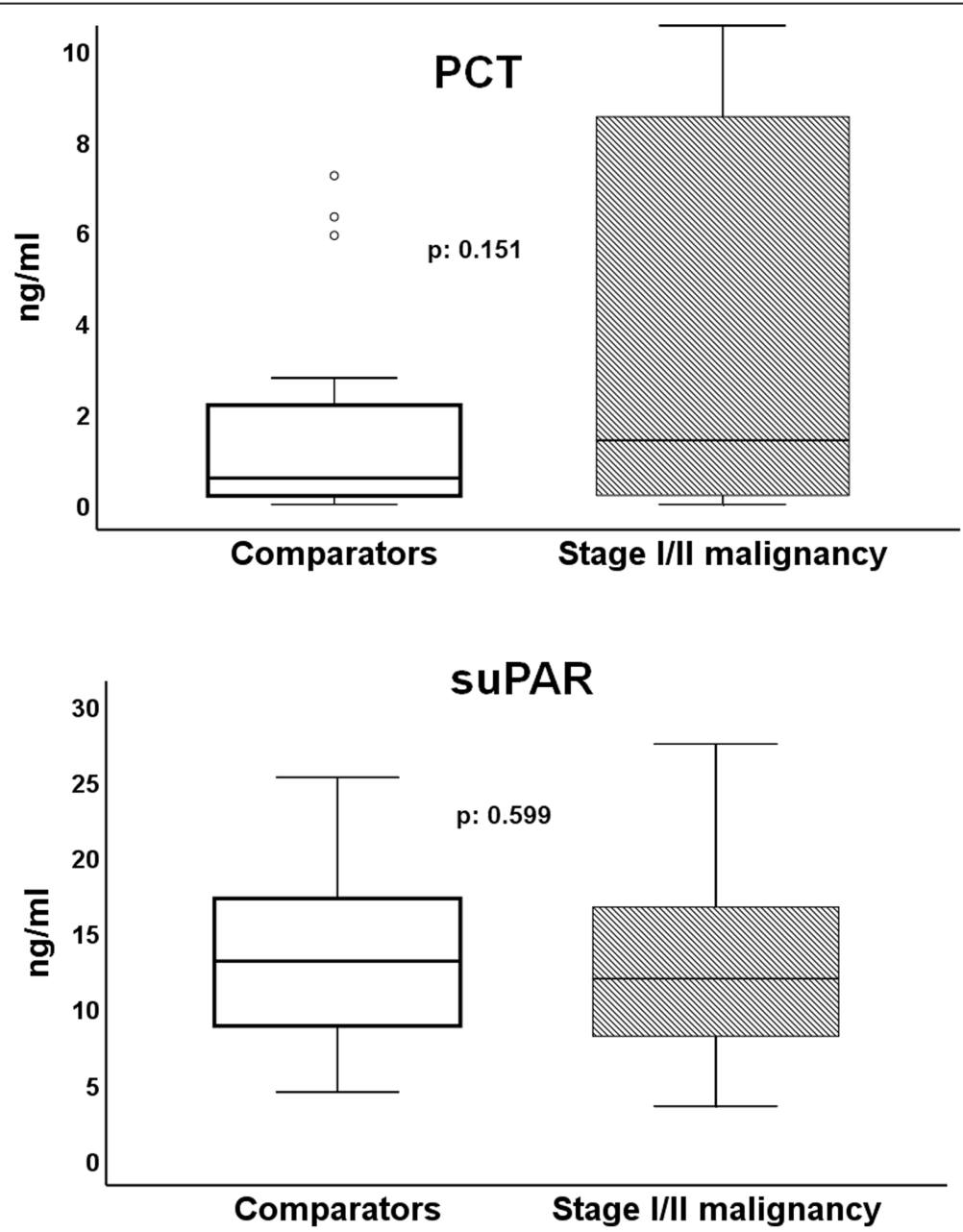

Fig. 3 Concentrations of procalcitonin (PCT) and of soluble urokinase plasminogen activator receptor (suPAR) of 83 patients with sepsis and history of stage I/II malignancy and of 83 well-matched sepsis comparators. The respective $p$-values of comparisons are provided 
unfavorable outcome [18], results presented here indicate that past history of malignancy cannot affect circulating levels more than the septic process.

Another interesting finding of the present study, although bizarre at first glance, is the protective role of coronary heart disease. It is not the first time where a similar finding is reported. An analysis of the National Inpatient Sample database on 7.1 million of hospitalizations for severe sepsis during the period 2007 to 2013 in the United States reported $0.15 \%$ incidence of cases of Tako-Scubo cardiomyopathy (TCC). TCC is characterized by hypokinesia, akinesia or dyskinesia of the left ventricle in the absence of coronary obstruction. TCC was an independent protective factor from 28-day mortality [19]. The low number of the studied patients, probably influencing the power of the results, signifies that this finding should be interpreted with caution.

\section{Conclusions}

The present study revealed that past history of stage I/II solid malignancy is an independent risk factor for 28-day mortality in sepsis. Results suggest that past history of stage I/II malignancy should be a variable taken into consideration in the endpoint analysis of sepsis trials.

\section{Abbreviations}

APACHE: Acute physiology and chronic health evaluation; CCI: Charlson's comorbidity index; Cl: Confidence interval; HSSG: Hellenic Sepsis Study group; ICU: Intensive care unit; OR: Odds ratio; PCT: Procalcitonin; SOFA: Sequential organ failure assessment; suPAR: Soluble urokinase plasminogen activator receptor; TCC: Tako-Scubo cardiomyopathy

\section{Acknowledgments}

None.

\section{Authors' contributions}

GD contributed to study design and analysis of data, drafted the manuscript and gave final approval of the version to be published. NR, MP, EA, DA, KV, GV and CR contributed to the enrolment of patients and collection of clinical data, drafted the manuscript and gave final approval of the version to be published. MK run statistical matching, drafted the manuscript and gave final approval of the version to be published. EJGB contributed to study design, analyzed the data, wrote the manuscript and gave final approval of the version to be published. It is ensured that all authors have read and approved the manuscript.

\section{Funding}

The study was funded by the Hellenic Institute for the Study of Sepsis. The funding source did not have any role in the design of the study and collection, analysis, and interpretation of data and in writing the manuscript.

\section{Availability of data and materials}

Data are available upon request. Please contact the corresponding author $\mathrm{E}$. J. Giamarellos-Bourboulis.

\section{Ethics approval and consent to participate}

The protocol was approved by the following Ethics Committees:

- Ethics Committee of ATTIKON University General Hospital of Athens

- Ethics Committee of Asklipieion General Hospital of Voula, Territory of

Athens

- Ethics Committee of "Center for Trauma Resuscitation- KAT" General

Hospital of Athens

- Ethics Committee of "Evangelismos" General Hospital of Athens

- Ethics Committee of "Evgenideio" Hospital of Athens
- Ethics committee of "Ippokrateion" General Hospital of Athens - Ethics Committee of "G. Gennimatas" General Hospital of Athens - Ethics Committee of "Laikon" General Hospital of Athens

- Ethics Committee of "Konstantopouleio-Aghia Olga" General Hospital of

Athens

- Ethics Committee of "Korgialeneion-Benakion" General Hospital of Athens

- Ethics Committee of "Sotiria" Athens General Hospital

- Ethics Committee of "Thriasio" Elefsis General Hospital

- Ethics Committee of "Aghios Dimitrios" General Hospital of Thessaloniki

- Ethics Committee of "G. Gennimatas" General Hospital of Thessaloniki

- Ethics Committee of "Aghios Pavlos" General Hospital of Thessaloniki

- Ethics Committee of "Theagenio" Hospital of Thessaloniki

- Ethics Committee of "Tzaneio" General Hospital of Piraeus

- Ethics committee of University General Hospital of Alexandroupolis

- Ethics Committee of University General Hospital of loannina

- Ethics Committee of General Hospital of Korinthos

- Ethics Committee of General Hospital of Ptolemaida

The patients were enrolled after written consent of themselves or their legal representative in the case of patients unable to consent.

\section{Competing interests}

George Dimopoulos is an editorial board member for BMC Infectious Diseases.

Evangelos J Giamarellos-Bourboulis has received honoraria (paid to the University of Athens) from AbbVie USA, Abbott CH, Biotest Germany, Brahms $\mathrm{GmbH}$, InflaRx GmbH, MSD Greece, Pfizer Greece and XBiotech Inc. He has received independent educational grants from AbbVie, Abbott, Astellas Pharma, AxisShield, bioMérieux Inc., Inflammatix Inc., InflaRx GmbH, the Medicines Company and XBiotech Inc. He has received funding from the FrameWork 7 program HemoSpec and from the Horizon2020 Marie-Curie project European Sepsis Academy (granted to the National and Kapodistrian University of Athens).

The other authors declare that they have no competing interests.

\section{Author details}

'2nd Department of Critical Care Medicine, National and Kapodistrian University of Athens, Medical School, Athens, Greece. ${ }^{2}$ Intensive Care Unit, 1st Department of Pulmonary Medicine, National and Kapodistrian University of Athens, Medical School, Athens, Greece. IIntensive Care Unit, Korgialeneion-Benakeion Athens General Hospital, Athens, Greece. ${ }^{4}$ Intensive Care Unit, "G.Gennimatas" Thessaloniki General Hospital, Thessaloniki, Greece. ${ }^{5}$ Intensive Care Unit, Theageneion General Hospital, Thessaloniki, Greece. ${ }^{6}$ Intensive Care Unit, "Aghios Dimitrios" Thessaloniki General Hospital, Thessaloniki, Greece. ${ }^{7} 4$ th Department of Internal Medicine, National and Kapodistrian University of Athens, Medical School, Athens, Greece. ${ }^{8} 1$ st Department of Critical Care Medicine, National and Kapodistrian University of Athens, Medical School, Athens, Greece. ${ }^{9} 4$ th Department of Internal Medicine, ATTIKON University Hospital, 1 Rimini Street, 12462 Athens, Greece.

Received: 13 June 2019 Accepted: 10 September 2019

Published online: 07 October 2019

\section{References}

1. Azoulay E, Pochard F, Chevret S, Vinsonneau C, Garrouste M, Cohen Y, et al. Compliance with triage to intensive care recommendations. Crit Care Med. 2001;29:2132-6.

2. Peigne V, Rusinova K, Karlin L, Darmon M, Fermand JP, Schlemmer B, et al. Continued survival gains in recent years among critically ill myeloma patients. Intensive Care Med. 2008:35:512-8.

3. Azoulay E, Alberti C, Bornstain C, Leleu G, Moreau D, Recher C, et al. Improved survival in cancer patients requiring mechanical ventilatory support: impact of noninvasive mechanical ventilatory support. Crit Care Med. 2001;29:519-25.

4. Taccone FS, Artigas AA, Sprung CL, Moreno R, Sakr Y, Vincent JL. Characteristics and outcomes of cancer patients in European ICUs. Crit Care. 2009;13:R15

5. Soares M, Caruso P, Silva E, Teles JM, Lobo SM, Friedman G, et al. Characteristics and outcomes of patients with cancer requiring admission to intensive care units: a prospective multicenter study. Crit Care Med. 2010;38:9-15. 
6. Kostakou E, Rovina N, Kyriakopoulou M, Koulouris NG, Koutsoukou A. Critically ill cancer patient in intensive care unit: issues that arise. J Crit Care. 2014;29:817-22.

7. Williams MD, Braun LA, Cooper LM, Johnston J, Weiss RV, Qualy RL, et al. Hospitalized cancer patients with severe sepsis: analysis of incidence, mortality, and associated costs of care. Crit Care. 2004:8:R291-8.

8. Giamarellos-Bourboulis EJ, Tsaganos T, Tsangaris I, Lada M, Routsi C, Sinapidis $D$, et al. Validation of the new sepsis-3 definitions: proposal for improvement in early risk identification. Clin Microbiol Infect. 2017;23:104-9.

9. Groeger JS, Lemeshow S, Price K, Nierman DM, White P Jr, Klar J, et al. Multicenter outcome study of cancer patients admitted to the intensive care unit: a probability of mortality model. J Clin Oncol. 1998;16:761-70.

10. Ferra C, Marcos P, Misis M, Morgades M, Bordejé ML, Oriol A, et al. Outcome and prognostic factors in patients with hematologic malignancies admitted to the intensive care unit: a single-center experience. Int J Hematol. 2007;85: 195-202.

11. Sims SM, Kao AM, Spaniolas K, Celio AC, Sippey M, Heniford BT, et al. Chronic immunosuppressant use in colorectal cancer patients worsens postoperative morbidity and mortality through septic complications in a propensity-matched analysis. Colorectal Dis. 2019;21:156-63.

12. Lai CC, Ho CH, Chen CM, Chiang SR, Chao CM, Liu WL, et al. Risk factors and mortality of adults with lung cancer admitted to the intensive care unit. J Thorac Dis. 2018;10:4118-26.

13. Zhang Q, Zhang W, Li Z, Bai C, Li D, Zheng S, et al. Bacteremia due to AmpC $\beta$-lactamase producing Escherichia coli in hospitalized cancer patients: risk factors, antibiotic therapy, and outcomes. Diagn Microbiol Infect Dis. 2017:88:247-51.

14. Islas-Muñoz B, Volkow-Fernández P, Ibanes-Gutiérrez C, Villamar-Ramírez A, Vilar-Compte D, Cornejo-Juárez P. Bloodstream infections in cancer patients. Risk factors associated with mortality. Int J Infect Dis. 2018;17:59-64.

15. Dagher GA, El Khuri C, Chehadeh AAH, Chami A, Bachir R, Zebian D, et al. Are patients with cancer with sepsis and bacteremia at a higher risk of mortality? A retrospective chart review of patients presenting to a tertiary care Centre in Lebanon. BMJ Open. 2017;7:e013502.

16. Moore JX, Akinyemiju T, Bartolucci A, Wang HE, Waterbor J, Griffin R. A prospective study of cancer survivors and risk of sepsis within the REGARDS cohort. Cancer Epidemiol. 2018;55:30-8.

17. Eugen-Olsen J, Giamarellos-Bourboulis EJ. suPAR: the unspecific marker for disease presence, severity and prognosis. Int J Antimicrob Agents. 2015; 46(Suppl):S33-4.

18. Chounta A, Ellinas C, Tzanetakou V, Pliarhopoulou F, Mplani V, Oikonomou $A$, et al. Serum soluble urokinase plasminogen activator receptor as a screening tool for the early diagnosis of hepatocellular carcinoma. Liver Int. 2015;35:601-7

19. Vallabhajosyula S, Deshmukh AJ, Kashani K, Prasad A, Sakhuja A. Tako-Tsubo cardiomyopathy in severe sepsis: nationwide trends, predictors, and outcomes. J Am Heart Assoc. 2018;7:e009160.

\section{Publisher's Note}

Springer Nature remains neutral with regard to jurisdictional claims in published maps and institutional affiliations.

Ready to submit your research? Choose BMC and benefit from:

- fast, convenient online submission

- thorough peer review by experienced researchers in your field

- rapid publication on acceptance

- support for research data, including large and complex data types

- gold Open Access which fosters wider collaboration and increased citations

- maximum visibility for your research: over $100 \mathrm{M}$ website views per year

At BMC, research is always in progress.

Learn more biomedcentral.com/submissions 